\title{
INTERNAL LARGE-SCALE TOROIDAL MAGNETIC FIELD OF THE SUN
}

\author{
V.N.KRIVODUBSKIJ \\ Kiev Shevchenko University \\ Astronomical Observatory \\ Observatornaya Street, 3 \\ Kiev - 53, 252053 \\ Ukraine, U.S.S.R.
}

The evolution of the large-scale toroidal magnetic field $B_{\varphi}$ of the Sun generating in the radiative zone by acts of differential rotation on the relict radial magnetic field $\mathrm{B}_{\mu}$ is investigated regarding the magnetic buoyancy and ohmic dissipation.

The magnetic buoyancy plays main role in the constraint of the magnetic induction amplitude of the toroidal magnetic field generated on the up-to-date stage of the evolution of the Sun.

The condition of the stationarity $\partial \mathrm{B} \varphi / \partial t=0$ yields the maximum estimation of the magnetic induction of toroidal field in the range $10^{7}-2 \times 10^{8} \mathrm{G}$ when the value of the radial gradient of the angular velocity near the core of the Sun is about $5 \times 10^{-16} \mathrm{rad} / \mathrm{s} \cdot \mathrm{sm}$ (which is infered from helioseismology investigations) and when the relict radial field induction is about $1 \times 10-3-5 \times 104 \mathrm{G}$. The characteristic time scales of reaching established stationary regime for these fields change in the range from 50 millions years to 16 years.

Toroidal magnetic fields due to energetic constraints must be concentrated in relative thin magnetic flux tubes with diameters $16,000-800 \mathrm{~km}$ and with magnetic fluxes $2 \times 10^{25}-1 \times 10^{24} \mathrm{~N} \mathrm{x}$.

E. R. Priest and V. Krishan (eds.), Basic Plasma Processes on the Sun, 57. (C) 1990 IAU. Printed in the Netherlands. 